\title{
Data mining for dwarf novae in SDSS, GALEX and astrometric catalogues
}

\author{
Patrick Wils, ${ }^{1 \star}$ Boris T. Gänsicke, ${ }^{2}$ Andrew J. Drake ${ }^{3}$ and John Southworth ${ }^{2}$ \\ ${ }^{1}$ Vereniging voor Sterrenkunde, Belgium \\ ${ }^{2}$ Department of Physics, University of Warwick, Coventry CV4 7AL \\ ${ }^{3}$ California Institute of Technology, 1200 E. California Blvd, Pasadena, CA 91125, USA
}

Accepted 2009 October 16. Received 2009 October 16; in original form 2009 September 1

\begin{abstract}
By cross-matching blue objects from the Sloan Digital Sky Survey with Galaxy Evolution Explorer and the astrometric catalogues USNO-B1.0, GSC2.3 and CMC14, 64 new dwarf nova candidates with one or more observed outbursts have been identified. 14 of these systems are confirmed as cataclysmic variables through existing and follow-up spectroscopy. A study of the amplitude distribution and an estimate of the outburst frequency of these new dwarf novae and those discovered by the Catalina Real-time Transient Survey indicate that besides systems that are faint because they are farther away, there also exists a population of intrinsically faint dwarf novae with rare outbursts.
\end{abstract}

Key words: stars: dwarf novae - novae, cataclysmic variables.

\section{INTRODUCTION}

One of the astrophysically interesting evolutionary phases a close binary star can go through is that of a cataclysmic variable $(\mathrm{CV})$, in which a main-sequence star filling its Roche lobe loses mass to a white dwarf. In the case the white dwarf has no or only a weak magnetic field, an accretion disc forms around it. Because of thermal instabilities in the accretion disc some of these systems, the dwarf novae, occasionally outburst and become several magnitudes brighter for a few days to a few weeks at most (Meyer \& MeyerHofmeister 1984; Osaki 1996; Lasota 2001). Theoretical studies of the orbital period distribution of these dwarf novae predicted a large number of systems with an orbital period near the period minimum (Kolb 1993; Howell, Nelson \& Rappaport 2001). Until recently, the predicted accumulation of CVs near the period minimum was not what was observed (Kolb \& Baraffe 1999; Willems et al. 2005). Furthermore, there is a discrepancy between the theoretical period minimum of $\approx 70 \mathrm{~min}$ and the observed one between 80 and $86 \mathrm{~min}$ (Kolb \& Baraffe 1999). The Sloan Digital Sky Survey (SDSS) has led to the spectroscopic identification of a large sample of faint CVs (see Szkody et al. 2009, and earlier papers in the series). Follow-up studies reveal that this faint SDSS CV population differs in many aspects from the previously known brighter systems, and that its period distribution is in closer agreement with the theoretical predictions (Gänsicke et al. 2009), although the discrepancy between the observed and the theoretically predicted orbital period minimum remains. Also the observed ratio of short to long-period CVs still disagrees with the model predictions (Aungwerojwit et al. 2006; Pretorius, Knigge \& Kolb 2007; Pretorius \& Knigge 2008). While
SDSS has made already a substantial contribution to observational population studies of CVs, the completeness of the currently known CV sample remains rather uncertain.

Prior to SDSS, a large fraction of all CVs in general, and the majority of dwarf novae in particular, have been discovered serendipitously by their large amplitude variability, favouring those systems with frequent outbursts (Gänsicke 2005). The majority of dwarf novae are expected to have evolved to short periods near the period minimum, and to have a low mass transfer rate. Consequently, they have infrequent outbursts, and the probability for a serendipitous discovery is lower than for systems with longer orbital periods and higher mass transfer rates. Historically, a large fraction of these serendipitous discoveries were made by amateur observers, which implies that the current sample of dwarf novae is biased towards relatively bright objects. In addition, it is very difficult to assess the spatial and temporal coverage of their searches for transient objects. The Catalina Real-time Transient Survey ${ }^{1}$ (CRTS; Drake et al. 2009), based on data from the Catalina Sky Survey (CSS), is now routinely discovering dwarf novae. Because this is a much more systematic survey, which also goes to fainter magnitudes, it is expected that it will eventually provide strong constraints on the total number and the magnitude distribution of dwarf novae.

Here, we cross-match a number of large surveys to find faint outbursting dwarf novae, and make use of CRTS light curves to compare the properties of the previously known dwarf novae, those identified spectroscopically by SDSS, and the ones discovered in this paper.

\footnotetext{
${ }^{\star}$ E-mail: patrickwils@yahoo.com
}

${ }^{1}$ http://voeventnet.caltech.edu/feeds/Catalina.shtml 
Table 1. Known dwarf novae with SDSS data. The full table is available in the electronic version of the article (see Supporting Information).

\begin{tabular}{|c|c|c|c|c|c|c|c|c|c|}
\hline Name & SDSS & Type & $P_{\text {orb }}$ & $r$ & $u-g$ & $g-r$ & $r-i$ & $i-z$ & Method \\
\hline SDSS J013132.39-090122.3 & J013132.39-090122.2 & $\mathrm{UG}+\mathrm{ZZ}$ & 1.36 & 18.42 & 0.02 & -0.12 & -0.18 & 0.13 & \\
\hline SDSS J015151.87+140047.2 & J015151.87+140047.2 & UG & 1.98 & 20.00 & -0.29 & 0.30 & 0.41 & 0.45 & \\
\hline SDSS J031051.66-075500.3 & J031051.66-075500.3 & UGSU & 1.60 & $15.74 *$ & 0.26 & -0.27 & -0.16 & -0.22 & $\mathrm{D}$ \\
\hline CSS081107:033104+172540 & J033104.44+172540.2 & UG & & 19.33 & 0.09 & 0.54 & 0.45 & 0.41 & $\mathrm{D}$ \\
\hline CSS080303:073921+222454 & J073921.17+222453.5 & UG & & 22.40 & 0.06 & 0.27 & 0.27 & -0.37 & \\
\hline- & - & - & & & - & & & & \\
\hline
\end{tabular}

Note. An asterisk after the $r$ magnitude indicates the object was in outburst at the time of the SDSS photometry. The dwarf nova subtypes are defined as follows: UG: U Gem without subclassification; UGSS: SS Cyg subtype; UGZ: Z Cam subtype; UGSU: SU UMa subtype, UGWZ: WZ Sge subtype; E: eclipsing; ZZ: ZZ Ceti type white dwarf pulsations. The orbital period, taken from the online catalogue of Ritter \& Kolb (2003), is given in hours. The recovery method refers to the four substeps from Fig. 2: A (match with GALEX), B (SDSS variability), C (no USNO counterpart) and D (matching with astrometric catalogues).

\section{SEARCH FOR DWARF NOVAE}

To constrain the colours of candidate dwarf novae, SDSS photometry was obtained for a list of known dwarf novae taken from the AAVSO Variable Star Index. ${ }^{2}$ A sample of 163 objects was selected, presented in Table 1. Excluding outliers, this led to the following conditions:

$u-g<0.9, \quad g-r<0.8, \quad r-i<0.8, \quad i-z<1.0$.

Lower limits on the colours were not deemed necessary. The cut-offs for the $u-g$ and $g-r$ colours are illustrated in Fig. 1 .

A summary of the search procedure is given as a flow chart inFig. 2. Our initial master list of objects are all point sources within SDSS Data Release 7 (DR7; Abazajian et al. 2009) satisfying equation (1). These objects were then matched to repeat observations within SDSS and to Galaxy Evolution Explorer (GALEX) objects (Martin et al. 2005), adopting a search radius of 1 arcsec. For this, the predefined cross-matching tables in SDSS and GALEX CasJobs (Li \& Thakar 2008) and Budavári et al. (2009) were used.

The master list was also matched to the entries in the astrometric catalogues USNO-B1.0 (Monet et al. 2003), GSC2.3 (Lasker et al. 2008) and CMC14 (Copenhagen University Obs. et al. 2006), using VizieR (Ochsenbein, Bauer \& Marcout 2000). In this case, a matching radius of 2 arcsec was used for USNO-B1.0 and GSC2.3, and 1 arcsec for CMC14, but in most cases the resulting matches were much closer than this radius. To further limit the number of candidates to match against these astrometric catalogues, the following more stringent condition on the colours was applied:

$(u-g)+0.85(g-r)<0.18$.

This condition was found by optimizing the number of known dwarf novae kept in the sample, while 90 per cent of the blue stars were excluded. Condition (2) still retained more than 60 per cent of the original sample of 163 objects, and thus maintained the best chances to find new dwarf novae, while the amount of work involved in checking candidates was reduced tenfold. A bias towards short period dwarf novae is, however, introduced in this way: of the systems with an orbital period below the period gap 73 per cent (58 out of 79) satisfy condition (2), while only 31 per cent (five out of 16) of the systems above the period gap do. This can be

\footnotetext{
2 http://www.aavso.org/vsx
}

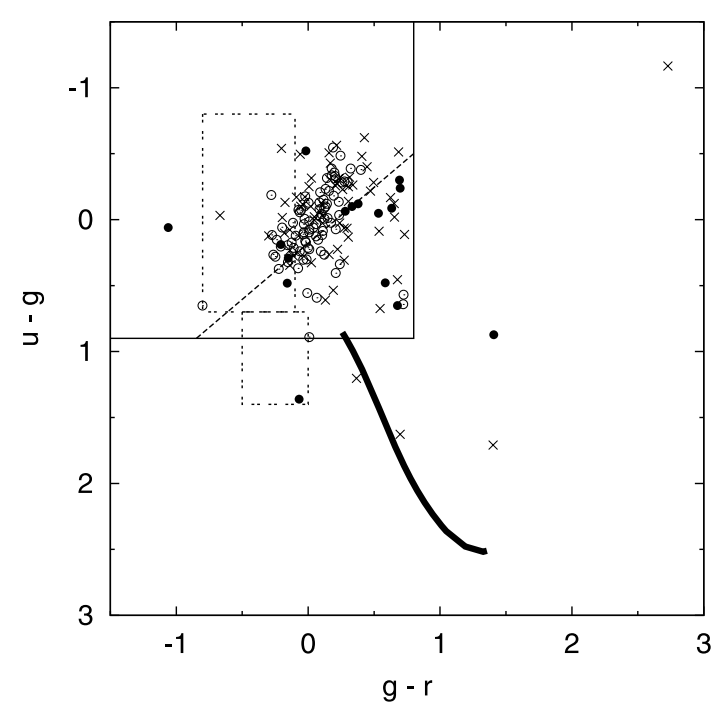

Figure 1. SDSS colours for a sample of 163 known dwarf novae. Open circles represent systems with an orbital period below the period gap, filled circles those with a period above the period gap. Crosses indicate the systems for which the orbital period is not known. The full lines denote the cut-offs used for selecting candidate dwarf novae. For matching with astrometric catalogues, candidates needed to lie above the dashed line as well. The curved line represents the stellar locus as determined by Richards et al. (2002). For illustration purposes only, the exclusion boxes for white dwarfs (top) and A stars (bottom) from Richards et al. (2002) are drawn with dotted lines. The outlier point in the top-right corner is for CSS090102:132536+210037. It is possible that the object entered an eclipse during the time SDSS cycled through the different passbands.

explained by the fact that short period systems are mostly white dwarf dominated, while in longer period systems the red dwarf will be larger, and therefore the combined colour will be redder. In the $(u-g, g-r)$ diagram, most CVs seem to cluster around a line with a slope similar to the one in condition (2) and almost perpendicular to the main sequence (see Fig. 1 and also fig. 6 from Gänsicke et al. 2009).

Objects were then selected as candidate transients when $|n u v-u|$ $\geq 2$ for the GALEX cross-matches (method 'A'), or when all of the ugriz magnitudes differed by at least $1.5 \mathrm{mag}$ in the case of multiple SDSS imaging runs (method 'B'), or when it had no counterpart in the USNO cross-matching table from SDSS CASJoBs (method ' $\mathrm{C}$ '). 


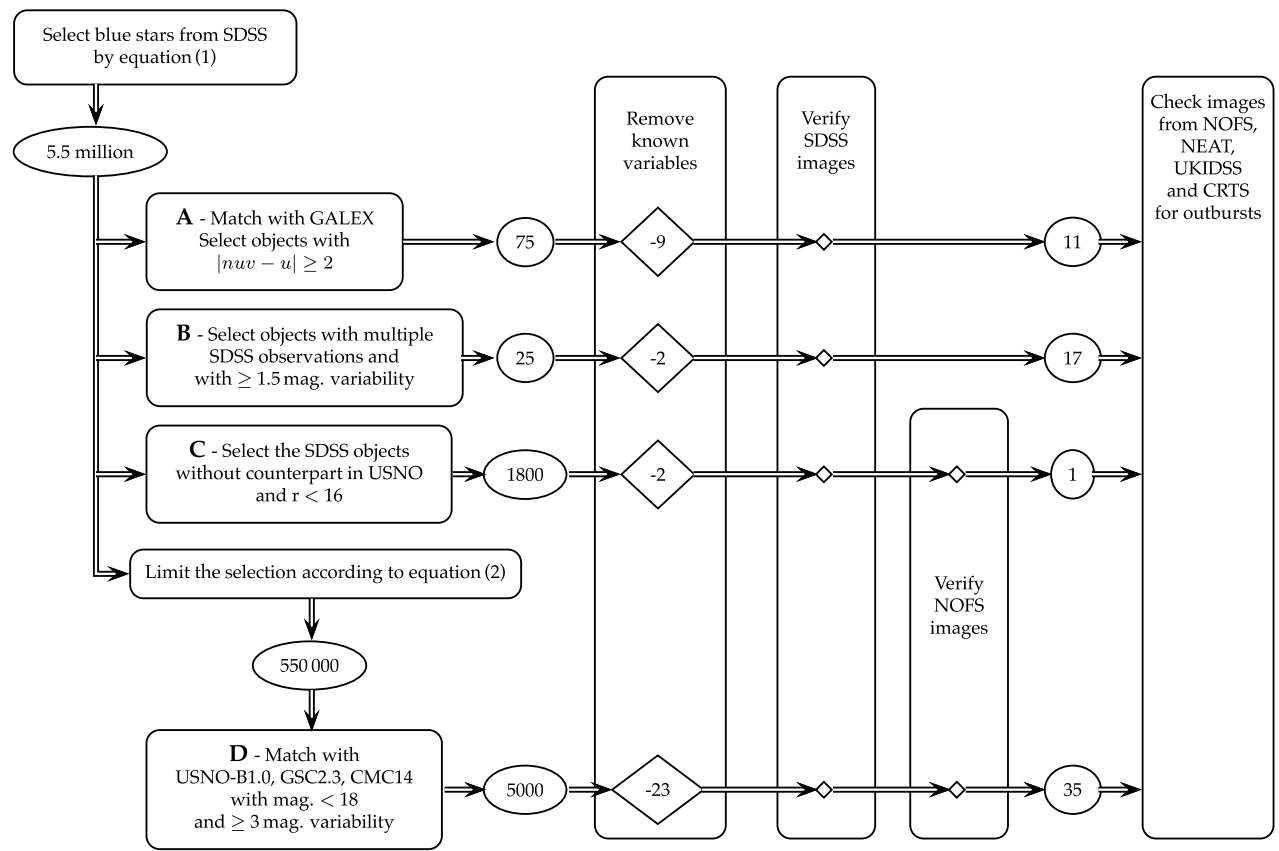

Figure 2. Schematic presentation of the search procedure. Numbers in ellipses indicate the number of objects selected in the previous step. Bold capital letters A-D indicate the different methods explained in detail in the text.

Further candidates were selected from objects that had an entry brighter than mag 18 in one of the astrometric catalogues, with at least a 3 mag difference with respect to the SDSS $r$ value (method 'D'). The reason for the latter constraint is that because USNOB1.0 and GSC2.3 are constructed from single images at a given epoch, possible outbursts cannot be confirmed on a second image from around that time. Given the limiting magnitude of about 20 for these catalogues, outburst detections fainter than mag 18 might be questionable. Since dwarf novae in outburst are nearly white $(B-$ $V \approx 0$ ), no colour correction was made for the filters used to obtain the catalogue magnitudes.

In this way, some 6500 transient candidates remained in total (taking into account that some candidates were selected by different methods). Among them, 36 known dwarf novae from the original sample in Table 1 were recovered. The repartition among the different methods is given in Fig. 2. The recovered sample viewed as a whole contained 22 per cent of the original sample of systems below the period gap, and 19 per cent of the systems above the period gap. For method 'D' only, these fractions are respectively 14 and 6 per cent. So, although this method is biased towards systems below the period gap, this bias is reflected less in the total number of recovered objects. The 36 known dwarf novae consisted of 15 confirmed SU UMa stars, one WZ Sge star (AL Com) and also seven dwarf novae recently discovered by CRTS. An outburst to $r=13.7$ (CMC14, 2003 April) of the suspected large amplitude eruptive variable EL UMa was also found, confirming it to be a dwarf nova. The first outburst of this system was detected by Pesch \& Sanduleak (1987) in 1981 May. In addition to those 36 known dwarf novae, also the nova CT Ser was recovered. The USNO-B1.0 B1 and R1 images date from 1950 June, about 2.5 years after its 1948 outburst which reached at least magnitude of 8.5 (Lohmann 1949). At the time of the images, CT Ser was still brighter (around mag 13) than in quiescence (mag 16).

SDSS images were then inspected to make sure that the remaining candidates were indeed stars, since a large fraction of the se- lected objects turned out to be parts of galaxies, or foreground stars to galaxies. In the latter case, the photometry in the astrometric catalogues cannot be trusted. Also some stars in the glare of a nearby bright star were excluded. The candidates were then examined on images of the United States Naval Observatory, Flagstaff Station (NOFS) ${ }^{3}$ to exclude image artefacts in the compilation of the USNO-B1.0 and GSC2.3 catalogues. Images of the Near Earth Asteroid Tracking (NEAT) ${ }^{4}$ survey were also examined to confirm the variability of some of the dubious candidates, and to look for additional outbursts of the real transients. Two possible outbursts were also found in data of the United Kingdom Infrared Telescope Infrared Deep Sky Survey (UKIDSS; Lawrence et al. 2007; Warren et al. 2007). As a large number of images were inspected visually, we do not claim that this search is exhaustive. Finally, for the remaining real transients, photometric data from CRTS were collected (dating back to late 2004), resulting in the detection of additional outbursts. ${ }^{5}$ One of the reasons the CRTS system did not trigger a transient alert when some of these objects were observed in outburst was that the CRTS pipeline compares its photometric data with SDSS and USNO-B1.0. When a dwarf nova has been observed in outburst in one of these surveys, it cannot reach the threshold required for a transient detection.

As a result, a total of 64 new candidate dwarf novae were found, which are listed in Table 2 together with their SDSS magnitudes. Table 3 gives a list of the observed outbursts, where an outburst is defined as an observation to within 1 mag of the brightest detection from Table 2.

There is always the possibility that some of the outbursting dwarf novae candidates found in this study are in reality optically violent

\footnotetext{
${ }^{3}$ http://www.nofs.navy.mil/data/fchpix/

${ }^{4}$ http://skyview.gsfc.nasa.gov/skymorph/skymorph.html

${ }^{5}$ The CRTS light curves compiled for this analysis are made available at http://nesssi.cacr.caltech.edu/catalina/20010604/
} 
Table 2. Candidate and confirmed dwarf novae.

\begin{tabular}{|c|c|c|c|c|c|c|c|c|c|c|}
\hline SDSS & fuv & nuv & $u$ & $g$ & $r$ & $i$ & $z$ & Mag range & Method & Spectrum \\
\hline J015237.83-172019.3 & & & 21.43 & 21.38 & 21.43 & 21.30 & 20.99 & $16.0-21.4$ & $\mathrm{D}$ & \\
\hline J064911.48+102322.1 & & & $17.61^{*}$ & 17.26 & 17.20 & 17.24 & 17.29 & $17.2-18.9$ & B & \\
\hline J073208.11+413008.7 & 17.07 & $17.09^{*}$ & 20.45 & 20.77 & 20.44 & 20.23 & 19.84 & $15.6-20.4$ & $\mathrm{~B}$ & SDSS $(g=20.7)$ \\
\hline J073758.55+205544.5 & 20.24 & 20.01 & $17.19^{*}$ & 17.39 & 17.23 & 17.28 & 17.27 & $17.2-20.0$ & $\mathrm{~B}$ & \\
\hline $\mathrm{J} 075107.50+300628.4$ & 19.02 & 19.62 & 19.57 & 19.76 & 19.81 & 19.83 & 19.74 & $15.1-19.8$ & $\mathrm{D}$ & \\
\hline J075117.00+100016.2 & 18.70 & 18.35 & 17.93 & 18.52 & 18.39 & 18.41 & 18.33 & $14.5-18.4$ & $\mathrm{D}$ & \\
\hline J075713.81+222253.0 & 17.88 & $17.93^{*}$ & 20.98 & 21.26 & 21.18 & 21.06 & 21.40 & $17.0-21.2$ & $\mathrm{~A}$ & \\
\hline J080033.86+192416.5 & & & 19.55 & 19.74 & 19.72 & 19.71 & 19.58 & $15.9-19.7$ & $\mathrm{~B}$ & \\
\hline J080306.99+284855.8 & & 21.12 & $16.48^{*}$ & 16.28 & 16.42 & 16.58 & 16.70 & $15.8-20.4$ & $\mathrm{~B}$ & \\
\hline J081030.45+091111.7 & & & $19.74 *$ & 19.98 & 19.84 & 19.72 & 17.62 & $19.8-22.8$ & $\mathrm{~B}$ & \\
\hline J083508.99+600643.9 & & & 21.86 & 21.66 & 21.41 & 21.25 & 21.55 & $18.3-21.4$ & $\mathrm{~B}$ & \\
\hline J084011.95+244709.8 & & 22.14 & $19.07 *$ & 19.03 & 19.37 & 19.48 & 18.94 & $19.4-21.2$ & B & \\
\hline J084108.10+102536.2 & 20.90 & 21.12 & 19.85 & 20.24 & 20.14 & 20.00 & 20.08 & $15.8-20.1$ & $\mathrm{D}$ & \\
\hline J091147.02+315101.8 & 20.80 & 20.69 & $15.35^{*}$ & 15.17 & 15.51 & 15.76 & 15.96 & $15.4-20.0$ & $\mathrm{D}$ & \\
\hline J091242.18+620940.1 & & 20.04 & 18.73 & 18.81 & 18.73 & 18.44 & 17.86 & $14.5-18.7$ & $\mathrm{D}$ & $\operatorname{SDSS}(g=19.5)$ \\
\hline J091741.29+073647.4 & & 22.20 & 21.77 & 21.51 & 21.39 & 21.51 & 21.15 & $18.2-21.4$ & $\mathrm{D}$ & \\
\hline J092620.42+034542.3 & 18.40 & $17.87 *$ & 19.67 & 19.91 & 19.80 & 19.80 & 19.86 & $17.0-19.8$ & A & \\
\hline J092809.84+071130.5 & 22.07 & 21.40 & $15.58^{*}$ & 15.28 & 15.47 & 15.67 & 15.84 & $15.5-21.0$ & $\mathrm{D}$ & SDSS $(g=14.7)$ \\
\hline J093946.03+065209.4 & & 22.21 & $18.00^{*}$ & 17.74 & 18.00 & 18.17 & 18.38 & $17.4-22.0$ & $\mathrm{~B}$ & SDSS $(g=17.7)$ \\
\hline J100243.11-024635.9 & & & 21.18 & 21.38 & 21.52 & 21.24 & 20.47 & $16.3-21.5$ & $\mathrm{D}$ & \\
\hline J100516.61+694136.5 & & & 19.80 & 19.40 & 18.92 & 18.60 & 18.26 & $17.9-21.0$ & $\mathrm{D}$ & SDSS $(g=18.4)$ \\
\hline $\mathrm{J} 105333.76+285033.6$ & 18.27 & $18.03 *$ & 20.23 & 20.16 & 19.79 & 19.60 & 19.44 & $17.2-20.6$ & A & \\
\hline J112003.40+663632.4 & 22.13 & 21.53 & $15.81^{*}$ & 15.62 & 15.90 & 16.12 & 16.32 & $15.9-21.0$ & $\mathrm{D}$ & SDSS $(g=21.1-18.2)$ \\
\hline $\mathrm{J} 141029.09+330706.2$ & & & 23.25 & 22.44 & 22.24 & 22.24 & 22.56 & $19.8-22.2$ & $\mathrm{~B}$ & \\
\hline $\mathrm{J} 142414.20+105759.8$ & & & $18.41^{*}$ & 16.45 & 15.95 & 16.86 & 16.41 & $15.9-19.0$ & $\mathrm{~B}$ & \\
\hline $\mathrm{J} 142953.56+073231.2$ & 21.65 & 21.19 & 21.00 & 21.21 & 20.80 & 20.32 & 19.81 & $16.9-20.8$ & $\mathrm{D}$ & \\
\hline $\mathrm{J} 151109.79+574100.3$ & 17.97 & $17.74 *$ & 20.89 & 21.24 & 21.18 & 21.04 & 20.88 & $17.3-21.2$ & A & \\
\hline $\mathrm{J} 152124.38+112551.9$ & & & $20.02 *$ & 19.40 & 19.28 & 19.28 & 19.16 & $19.3-21.7$ & $\mathrm{~B}$ & GMOS $(g=21.8)$ \\
\hline J153457.24+505616.8 & & 22.09 & 21.94 & 22.01 & 21.78 & 21.72 & 21.04 & $17.0-21.8$ & $\mathrm{D}$ & \\
\hline J154357.66+203942.1 & 22.36 & 21.25 & $16.93^{*}$ & 16.58 & 16.74 & 16.88 & 17.01 & $15.6-21.0$ & $\mathrm{D}$ & \\
\hline $\mathrm{J} 154652.70+375415.2$ & 16.39 & 16.46 & $16.01 *$ & 16.03 & 16.27 & 16.46 & 16.66 & $16.3-21.0$ & $\mathrm{D}$ & \\
\hline $\mathrm{J} 154817.56+153221.2$ & 17.51 & $17.33^{*}$ & 21.55 & 21.75 & 21.65 & 21.69 & 21.32 & $16.5-21.7$ & $\mathrm{~A}$ & \\
\hline $\mathrm{J} 155030.38-001417.3$ & 18.93 & 19.01 & 21.91 & 22.01 & 21.60 & 21.04 & 20.77 & $17.1-21.6$ & $\mathrm{~A}$ & \\
\hline $\mathrm{J} 155540.19+364643.1$ & 20.66 & 20.68 & 20.48 & 20.80 & 20.90 & 20.72 & 20.52 & $16.0-20.9$ & $\mathrm{D}$ & ACAM \\
\hline J161027.61+090738.4 & 20.95 & 20.65 & 20.19 & 20.10 & 20.06 & 20.26 & 20.03 & $15.0-20.1$ & $\mathrm{D}$ & SDSS $(g=20.2)$ \\
\hline J161442.43+080407.9 & & 23.41 & 21.44 & 21.24 & 21.41 & 20.95 & 21.35 & $16.8-21.4$ & $\mathrm{D}$ & \\
\hline $\mathrm{J} 162520.29+120308.7$ & 20.99 & 20.09 & 18.45 & 18.48 & 18.41 & 18.24 & 17.69 & $14.5-18.4$ & $\mathrm{D}$ & SDSS $(g=19.9)$ \\
\hline $\mathrm{J} 162558.18+364200.6$ & 21.73 & 22.04 & 21.49 & 21.68 & 21.60 & 21.54 & 21.89 & $17.1-21.6$ & $\mathrm{D}$ & \\
\hline $\mathrm{J} 162900.55+341022.0$ & 21.61 & 21.89 & 21.16 & 21.22 & 21.20 & 21.12 & 21.09 & $16.0-21.2$ & $\mathrm{D}$ & ACAM \\
\hline $\mathrm{J} 164705.07+193335.0$ & & & 21.91 & 21.95 & 21.79 & 21.68 & 21.34 & $16.7-21.8$ & $\mathrm{D}$ & \\
\hline $\mathrm{J} 170145.85+332339.5$ & & & 21.23 & 21.58 & 21.40 & 21.31 & 21.65 & $18.8-21.4$ & $\mathrm{~B}$ & GMOS $(g=22.2)$ \\
\hline $\mathrm{J} 170810.31+445450.7$ & 20.40 & 20.89 & 20.78 & 20.83 & 20.78 & 20.84 & 20.87 & $14.0-20.8$ & $\mathrm{D}$ & ACAM \\
\hline $\mathrm{J} 171202.95+275411.0$ & & & 21.24 & 21.39 & 20.94 & 20.51 & 20.58 & $17.3-20.9$ & $\mathrm{D}$ & $\operatorname{GMOS}(g=21.0)$ \\
\hline $\mathrm{J} 174839.77+502420.3$ & & & 23.24 & 22.30 & 23.02 & 23.78 & 24.15 & $15.3-23.0$ & $\mathrm{C}$ & \\
\hline
\end{tabular}


Table 2 - continued

\begin{tabular}{|c|c|c|c|c|c|c|c|c|c|c|}
\hline SDSS & fuv & nuv & $u$ & $g$ & $r$ & $i$ & $z$ & Mag range & Method & Spectrum \\
\hline J205931.86-070516.6 & 21.33 & 20.64 & 20.85 & 20.75 & 21.03 & 21.24 & 21.56 & $17.4-21.0$ & $\mathrm{D}$ & \\
\hline $\mathrm{J} 223854.51+053606.8$ & & 21.58 & 21.52 & 21.52 & 21.51 & 21.52 & 20.82 & $17.4-21.5$ & D & \\
\hline
\end{tabular}

Note. The SDSS magnitudes are from DR7 (Abazajian et al. 2009), ultraviolet magnitudes have been obtained from GALEX (Martin et al. 2005). An asterisk after the $n u v$ and the $u$ magnitude indicates that the GALEX and SDSS measurements were taken during outburst, respectively. The approximate observed amplitude of variability is provided in the $r$ band when available, otherwise the brightest and faintest observed magnitude are given, irrespective of passband. The discovery method refers to the four substeps from Fig. 2: A (match with GALEX), B (SDSS variability), C (no USNO counterpart) and D (matching with astrometric catalogues). Identification spectra are available for 16 objects, magnitudes at the time of the spectroscopy are provided where available.

Table 3. Observed outbursts of the new dwarf novae. The table is available in full in the electronic version of the article (see Supporting Information).

\begin{tabular}{cl}
\hline SDSS & Outbursts \\
\hline J013645.81-193949.1 & Oct 1997 (NOFS), Dec 2000 (NEAT), Nov 2004 (CMC14) \\
J015237.83-172019.3 & Sep 1978 (NOFS) \\
J032015.29+441059.3 & Nov 1994 (NOFS), Dec 2005 (SDSS) \\
J064911.48+102322.1 & Nov 2006 (SDSS) \\
J073208.11+413008.7 & Mar 1953 (NOFS), Dec 2001 (NEAT), Nov 2003 (SDSS), Feb 2007 (GALEX + CRTS) + 3 other outbursts (CRTS) \\
J073758.55+205544.5 & Jan 2002 (SDSS), Dec 2002 (NEAT), Feb 2006 (CRTS) \\
J074500.58+332859.6 & See the text \\
J074859.55+312512.6 & Dec 2001 (SDSS + NEAT), + 4 bright and 6 fainter outbursts (CRTS) \\
J075107.50+300628.4 & Mar 2003 (CMC14 + NEAT), Mar 2005 (CRTS), Jan 2006 (CRTS), Jan 2007 (CRTS), Dec 2007 (CRTS) \\
- & - \\
\hline
\end{tabular}

Note. Sources for the data are: Copenhagen University Obs. et al. (CMC14; 2006), Digitized Sky Survey, Drake et al. (CRTS; 2009), Martin et al. (GALEX; 2005), NEAT, United States NOFS, Ducourant et al. (PM2000; 2006), Abazajian et al. (SDSS; 2009) and Lawrence et al. (UKIDSS; 2007).

variable (OVV) quasars. Similar to dwarf novae, they can show outbursts up to 6 mag (Abraham \& Carrara 1998), but these outbursts generally last more than 3 months, much longer than a typical dwarf nova outburst. However, because of the limited data available for some of the outbursts from this paper, a distinction is not always possible. Unlike faint dwarf novae, OVV quasars and blazars in general are often also detected in radio wavelengths. None of the candidates dwarf novae presented in Table 2 is, however, known to have a radio counterpart. In addition, only three known quasars were found (SDSS J095315.74+441123.6, 2QZ J095551.3-020428 and SDSS J103705.22+463128.4, all by matching with astrometric catalogues), as opposed to 36 known dwarf novae.

\section{IDENTIFICATION SPECTROSCOPY}

Identification spectra are available for 16 of the 64 dwarf nova candidates: one from SDSS DR3 (Fig. 3; Abazajian et al. 2005), seven within SDSS DR7 (Fig. 4; Abazajian et al. 2009), and one from the 6dF Galaxy Survey (6dFGS) ${ }^{6}$ (Fig. 5; Jones et al. 2005, 2009). We obtained spectra for the other seven objects using GMOS on Gemini-North and ACAM on the William Herschel Telescope (Figs 6 and 7). Details of the GMOS and ACAM observations are briefly outlined in Section 3 before discussing details of individual systems in Section 4.

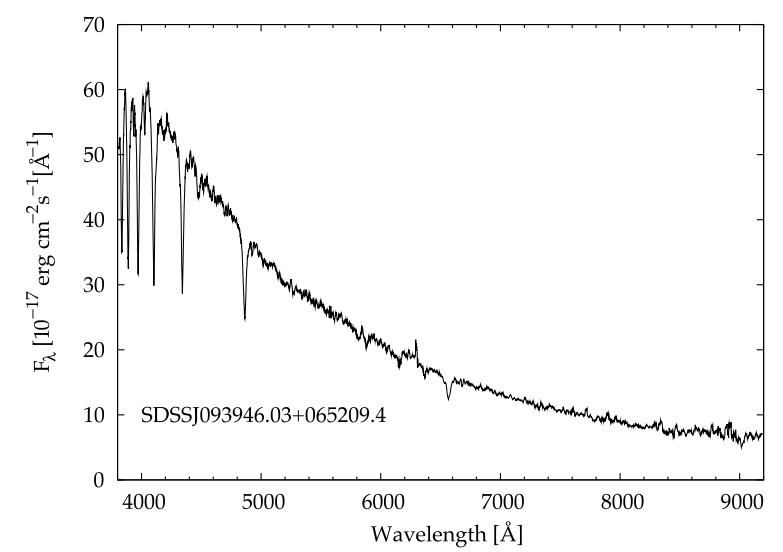

Figure 3. SDSS DR3 spectrum (Abazajian et al. 2005) of SDSS J093946.03+065209.4 in outburst.

\subsection{WHT/ACAM}

Spectroscopic observations were carried out in service mode in 2009 June, using the new $\mathrm{ACAM}^{7}$ spectrograph on the 4.2-m William Herschel Telescope (WHT). The default 400 lines $\mathrm{mm}^{-1}$ grism was used, giving a wavelength coverage of 4000-9000 $\AA$. The detector was an EEV $4096 \times 2048$ pixel CCD, binned by a factor of 2 in both dimensions to give a spatial resolution of $0.5 \operatorname{arcsec}$ pixel $^{-1}$ and a reciprocal dispersion of approximately $7 \AA \mathrm{pixel}^{-1}$ throughout the wavelength range. 


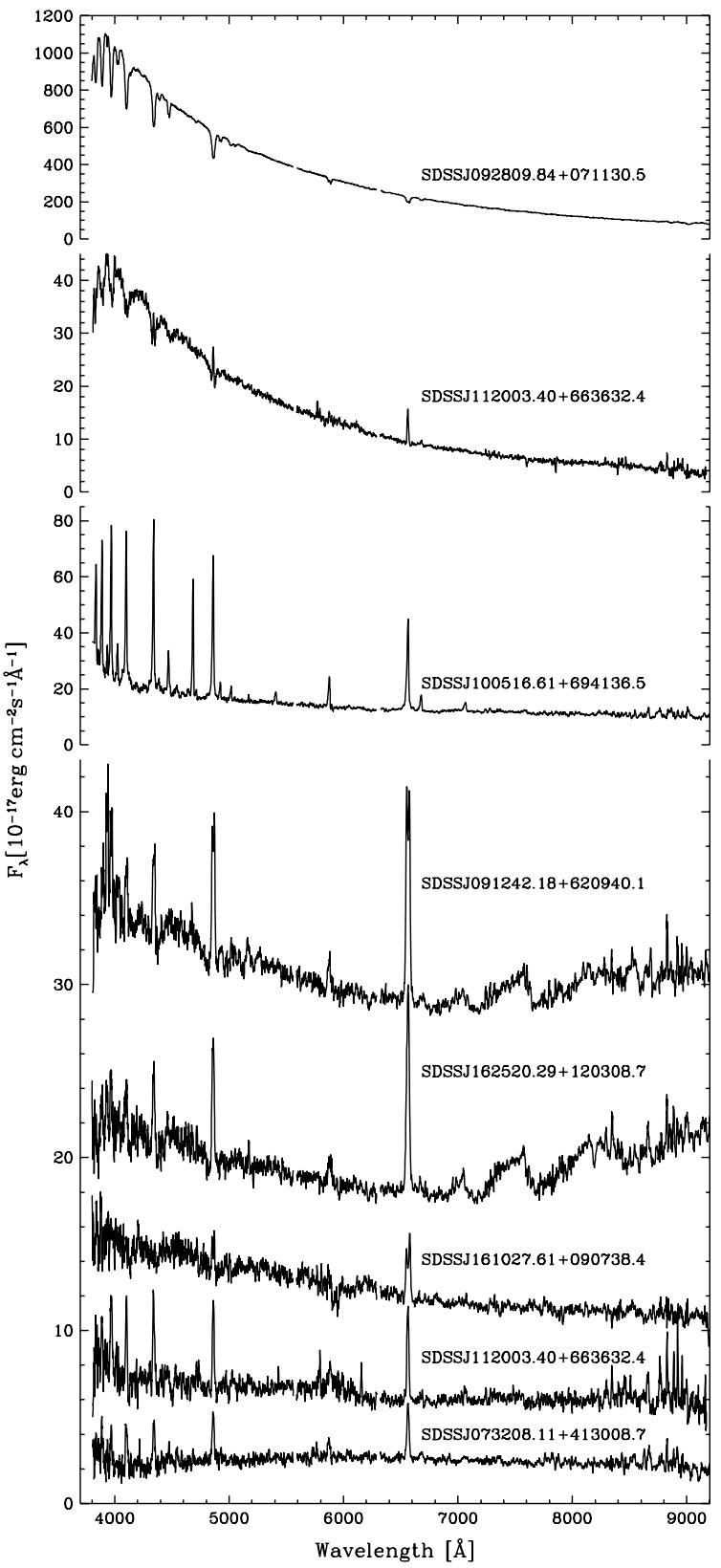

Figure 4. SDSS DR7 (Abazajian et al. 2009) spectra of seven CV candidates from Table 2. The spectra in the bottom panel are offset (top to bottom) by $25,15,10,5$ and 0 flux units. SDSS J112003.40+663632.4 was observed in quiescence (bottom panel) and on the decline from an outburst (second panel from top).

The data were reduced using optimal extraction (Horne 1986) as implemented in the PAMELA ${ }^{8}$ code (Marsh 1989). Wavelength calibration was performed using copper-argon and copper-neon arc lamp spectra taken immediately before or after the science observations, but is very approximate bluewards of $5850 \AA$ due to the lack of measurable emission lines in this region. From fitting Gaussian functions to emission lines from the night sky and from the arc lamps, we estimate the resolution of these spectra to be approximately $18 \AA$.

\footnotetext{
${ }^{8}$ PAMELA and MOLLY were written by TRM and can be found at
} http://www.warwick.ac.uk/go/trmarsh

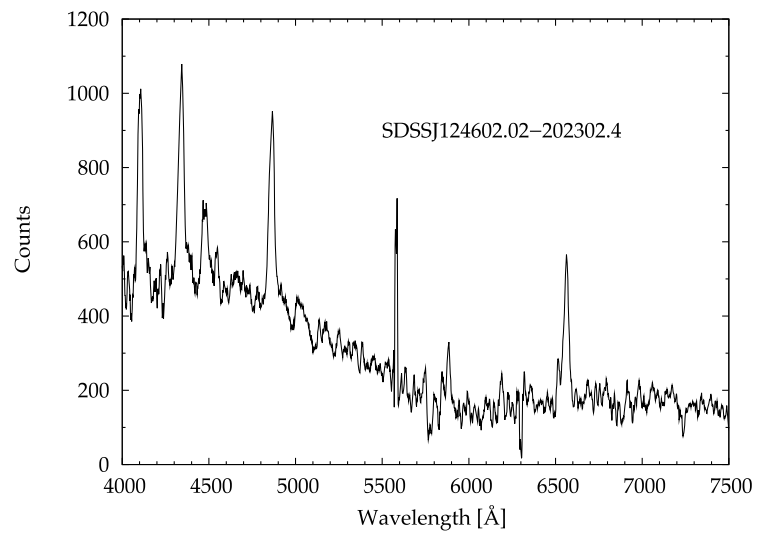

Figure 5. Spectrum of SDSS J124602.02-202302.4 from the 6dFGS (Jones et al. 2005, 2009).

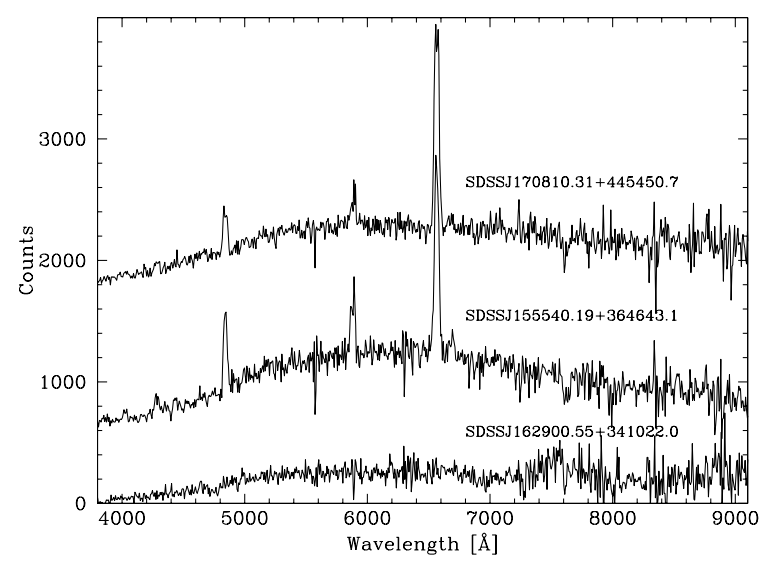

Figure 6. WHT/ACAM spectra for three of the new dwarf novae presented in this paper.

The reduced spectra (Fig. 6) were corrected for telluric absorption using spectra of the flux standard star BD $+26^{\circ} 2606$. Due to problems arising from the poor throughput at wavelengths below $\simeq 4500 \AA$, we decided not to apply any flux calibration.

\subsection{Gemini/GMOS}

Spectroscopic observations were performed with the Gemini-South telescope between 2008 June and 2009 April, with the GMOS-S instrument and the R150 grating. We used a central wavelength setting of $740 \mathrm{~nm}$ and a slit of width 1 arcsec. The detector of this spectrograph is a mosaic of three $2048 \times 4068$ pixel CCDs abutted on their long edges, and long-slit spectra are dispersed over all three CCDs. We reduced the spectra from CCD2 and CCD3 individually, in the same way as for the ACAM data, and ignoring CCD1 which covers the spectral region redwards of $9300 \AA$. The spectra were then flux calibrated using observations of LTT 6248 and the results from the different CCDs combined to form a summed spectrum for each object covering 3500-9200 ̊. Severe fringing is apparent redwards of $7000 \AA$. The wavelength calibration of CCD3 (3500$5550 \AA$ ) is only approximate due to the very small number of usable arc lines. The final spectra have a reciprocal dispersion of $3.5 \AA$ and a resolution of roughly $15 \AA$. The reduced spectra are shown in Fig. 7. 

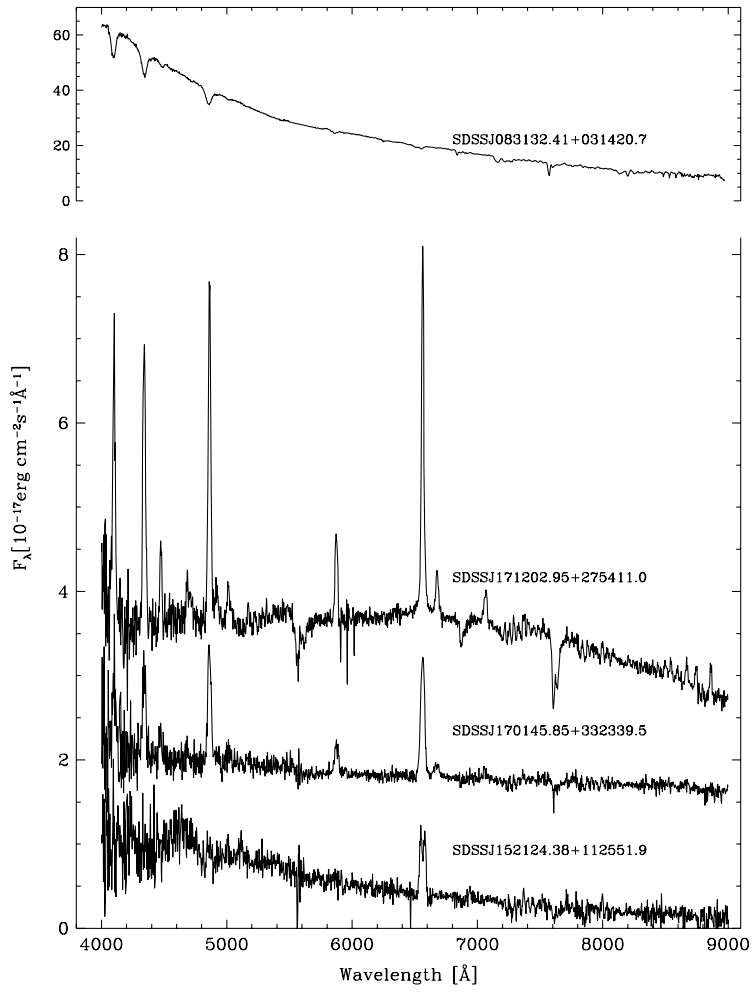

Figure 7. Gemini/GMOS spectra for four of the new dwarf novae presented in this paper. SDSS J083132.41+031420.7 was observed during outburst.

\section{DETAILS ON INDIVIDUAL SYSTEMS}

All the new systems with spectra, and some other systems with noteworthy aspects are briefly discussed in this section. Additional cross-identifications for objects from Table 2 which are listed in other catalogues, but were not yet identified as dwarf nova candidates, are given in Table 4.

\subsection{Systems with spectra}

Among the 16 systems for which spectroscopy has been obtained 13 have spectra consistent with being dwarf novae, one appears to be a polar or intermediate polar (SDSS J100516.61+694136.5), and two have spectra that are unusual for CVs (SDSS J093946.03+065209.4 and SDSS J162900.55+341022.0). SDSS J093946.03+065209.4 is the only pre-DR7 CV candidate that has not been listed by Szkody et al. (2009) and the previous papers of that series, for the reasons outlined below. The CVs with DR7 spectroscopy will be included in Szkody et al. (in preparation). (i) SDSS J073208.11+413008.7. The SDSS spectrum exhibits a rather red slope (Fig. 4), and one of the three SDSS photometric data sets shows a very unusual ugriz spectral energy distribution. The CRTS light curve of this object shows a high duty cycle, implying frequent outbursts. An alternative possibility is that the system is eclipsing.

(ii) SDSS J083132.41+031420.7. We observed this object with Gemini when it was in a bright state $(r \simeq 17.6)$. The spectrum is typical of a dwarf nova in outburst, with broad Balmer absorption lines from an optically thick accretion disc (Fig. 7).

(iii) SDSS J091242.18+620940.1. Both the white dwarf and the M-dwarf companion are visible in the SDSS spectrum (Fig.4), implying that this system has a low accretion rate.

(iv) SDSS J092809.84+071130.5. The SDSS spectrum was obtained during a bright state $(g=14.7)$, and is typical of a dwarf nova in outburst, similar to SDSS J083132.41+031420.7 (Fig. 4).

(v) SDSS J093946.03+065209.4. This object was classified as a subdwarf by Eisenstein et al. (2006). Its spectrum is actually more reminiscent of a star of spectral type A (Fig. 3), and is very unusual for a CV. This is probably also the reason why it was not included in the list of CVs from SDSS DR3 (Szkody et al. 2004). However, both NEAT and SDSS have it bright (around mag 18) the first half of 2002 March on two nights each, while it is faint (mag 22) on two nights in 2006 in SDSS, and invisible on NEAT images on 30 other nights, including images taken at the end of 2002 February. CRTS observed other outbursts to mag 17.4 (2005 May), mag 18.2 (2006 February), mag 19.1 (2008 January) and mag 17.8 (2008 June), but did not detect it most of the time either. The SDSS spectroscopic fluxes (from a year after the photometry) agree almost spot-on with the photometry, suggesting another outburst in 2003 March. This may be a CV with a very low inclination, where the Balmer lines from the accretion disc are only marginally Doppler broadened.

(vi) SDSS J100516.61+694136.5. With a strong He II 4686 line (Fig. 4), it is likely a magnetic CV, i.e. a polar or an intermediate polar. It is listed in the Two-Micron All-Sky Survey (2MASS) catalogue with $J=16.8$.

(vii) SDSS J112003.40+663632.4. The SDSS imaging found SDSS J1120+6636 at $r=15.9$ on MJD51661. SDSS spectroscopy of SDSS J1120+6636 was obtained on five different nights. On three occasions, the system was observed in quiescence at $r \simeq$ 21 (MJD 54464, 54483, 54485), and its spectrum exhibits narrow, moderately strong Balmer emission lines superimposed on a weak continuum (Fig. 4). Spectra obtained on MJD 54495 and 54498 showed the system on the decline from an outburst at $r \simeq 18.2$ and $\simeq 19.4$. The average SDSS spectrum available in DR7 is dominated by the data obtained on MJD 54495, and show a steep blue continuum with rather broad Balmer absorption lines, superimposed by weak emission lines with only the central core visible, as expected for an accretion disc in/close to outburst. CRTS detected a single additional outburst on MJD 53746.

Table 4. Cross-identifications for the new dwarf nova candidates.

\begin{tabular}{lll}
\hline SDSS & Other ID & Reference \\
\hline J092620.42+034542.3 & GUVV J092620.4+034541.8 & Welsh et al. (2005) \\
$\mathrm{J} 124328.27-055431.0$ & GUVV J124328.2-055431.7 & Welsh et al. (2005) \\
$\mathrm{J} 124602.02-202302.4$ & 6dFGS g1246019-202302 & Jones et al. (2005, 2009) \\
$\mathrm{J} 131432.10+444138.7$ & 2RXP J131432.9+444144 & \\
$\mathrm{J} 132040.96-030016.7$ & 1RXS J132041.2-030010 & Voges et al. (1999) \\
$\mathrm{J} 132715.28+425932.8$ & GUVV J132715.2+425932.1 & Welsh et al. (2005) \\
$\mathrm{J} 162900.55+341022.0$ & NSV 20657 & Hovanissian (1978, 1982) \\
\hline
\end{tabular}


(viii) SDSSJ124602.02-202302.4. The 6dFGS spectrum (Fig. 5) is reminiscent of a quiescent dwarf nova, with broad Balmer emission lines.

(ix) SDSS J152124.38+112551.9. This is an example of a CV with a white dwarf dominated optical spectrum and a steep Balmer line decrement (Fig. 7), resembling closely the class of intrinsically faint CVs with periods within the $80-86$ min period minimum spike that have been identified from SDSS spectroscopy (Gänsicke et al. 2009). The spectrum implies a low temperature in the accretion flow, which again most likely means a low accretion rate.

(x) SDSS J155540.19+364643.1. The ACAM spectrum (Fig. 6) is typical of a quiescent dwarf nova, with strong Balmer and $\mathrm{He}$ I emission lines.

(xi) SDSS J161027.61+090738.4. Based on the white dwarf dominated SDSS spectrum (Fig. 4), we suggest that this object is a WZ Sge type dwarf nova, with one outburst detected in 1998 June on NEAT images. While preparing the manuscript, SDSS 1610+0907 has entered a superoutburst, and follow-up photometry suggests a superhump period of $\simeq 82 \mathrm{~min}^{9}$

(xii) SDSS J162520.29+120308.7. The SDSS spectrum is dominated by emission from the white dwarf and the M-dwarf companion star, superimposed by broad emission lines (Fig. 4). The spectrum is similar to that of RZ Leo. The 2MASS catalogue lists the object at $J=16.6$ and $K_{s}=15.5$. The CRTS light curve is very unusual, exhibiting besides one outburst a modulation of the quiescent magnitude with a $\sim 1$ mag amplitude on time-scales of $\sim 500 \mathrm{~d}$. This is suggestive of stellar activity cycles on the companion star.

(xiii) SDSS J162900.55+341022.0. This is the only dwarf nova candidate where follow-up spectroscopy fails to detect Balmer emission lines (Fig. 6). The SDSS coordinates are consistent with those of NSV 20657, a bright blue object ( $B=17.9, U-B=-0.6$; Hovanissian 1978,1982 ) displaying variability with a 0.8 mag amplitude. The object is also listed in outburst in the USNO-B1.0 catalogue. The spectrum is consistent with that of a blazar, but the object does not have a radio counterpart. The untypical spectrum warrants additional observations.

(xiv) SDSS J170145.85+332339.5. The Gemini spectrum is typical of a quiescent dwarf nova (Fig. 7), with broad Balmer and He I emission lines.

(xv) SDSSJ170810.31+445450.7. The ACAM spectrum (Fig. 6) contains broad Balmer and He I emission lines, typical of a dwarf nova in quiescence.

(xvi) SDSSJ171202.95+275411.0. The Gemini spectrum (Fig. 7) shows strong Balmer and He I emission lines, typical of a quiescent dwarf nova.

\subsection{Systems without spectra}

(xvii) SDSS J064911.48+102322.1. The second SDSS observation was carried out only 2 days after the initial and brightest detection, so the system may actually have still been in the declining phase of the outburst rather than back in quiescence. In fact, the object was not found in other catalogues, suggesting that it is substantially fainter in quiescence.

(xviii) SDSS J074500.58+332859.5. The long-term light curve of this object is not consistent with being a dwarf nova. SDSS detected the object twice at $r \simeq 22.2$ on MJD 51874 and 51962, and once at $r=18.9$ on MJD 54138. CRTS monitoring started

\footnotetext{
${ }^{9}$ http://ooruri.kusastro.kyoto-u.ac.jp/pipermail/vsnet-alert/2009-
} August/002989.html around MJD 53319, but did not detect SDSS J0745+3328 until MJD $\simeq 53449$, when it reached $r \simeq 18.5$. The object stayed at that brightness level for $\simeq 600 \mathrm{~d}$, displaying short-term variability with a peak-to-peak magnitude of $\simeq 1 \mathrm{mag}$, and then faded over a period of $\simeq 400 \mathrm{~d}$ back to the CRTS detection threshold $\simeq 21$ over a period of $\simeq 400 \mathrm{~d}$. The long duration of both the high and low states, as well as the observed magnitude difference between the two states, is reminiscent of the long-term light curves of polars, and we speculate that SDSS J0745+3328 is a polar. Because it was not detected at radio wavelengths, it is not likely to be a blazar. SDSS J0745+3328 was not included in the further statistical analysis in Section 5.

(xix) SDSS J105333.76+285033.6. The CRTS light curves exhibits extremely frequent brightness variations, suggesting very frequent outbursts, or that the system is eclipsing.

(xx) SDSS J132040.96-030016.7. This system was found initially from cross-matches with GALEX by relaxing the conditions (1) and instead imposing $|f u v-n u v|<1$ in addition to a large UV excess. All the SDSS colours fit the conditions (1), except $g-$ $r=4.02$. As the sequence of filters in the SDSS photometry is $r, i$, $u, z, g$, it is possible that the white dwarf and/or the accretion disc was eclipsed by the red dwarf during this time. The source is also detected in the ROSAT All Sky Survey Bright Source Catalogue (Voges et al. 1999), making it particularly interesting for follow-up observations.

(xxi) SDSS J212025.17+194156.3. This object was also detected in outburst by CRTS during the preparation of this paper (2009 August).

\section{DISCUSSION}

For the remainder of the paper, we will work with all of the new dwarf novae found above as a single sample, irrespective of the method they were found with, as the individual subsamples are too small. As indicated above a bias towards short-period CVs was introduced by method ' $\mathrm{D}$ ', and this will be carried forward, although in a diminished form, into the complete sample.

Table 5 gives an overview of dwarf novae which have SDSS photometric data at minimum, divided into bins of 1 mag centred on the given $r$ value in the first column. The second column in the table gives the number of dwarf novae from our original sample (Table 1, excluding 19 objects which were only observed in outburst by SDSS). The third column gives the number of those systems that were not discovered by SDSS or CRTS, but were identified by other methods (mostly variability), and we will denote this

Table 5. Number of dwarf novae binned according to their $r$ magnitude in quiescence, based on the sample from Table 5 and the dwarf nova candidates in Table 2. See the text for details.

\begin{tabular}{crrrrrr}
\hline$r$ & Known & Old & SDSS & CRTS & Recovered & New \\
\hline 15 & 4 & 3 & 2 & 0 & 0 & 0 \\
16 & 9 & 8 & 6 & 1 & 2 & 0 \\
17 & 6 & 6 & 6 & 1 & 1 & 0 \\
18 & 31 & 13 & 27 & 9 & 3 & 4 \\
19 & 29 & 12 & 22 & 13 & 9 & 4 \\
20 & 26 & 11 & 4 & 13 & 6 & 11 \\
21 & 17 & 8 & 2 & 7 & 6 & 19 \\
22 & 15 & 2 & 1 & 15 & 1 & 14 \\
23 & 6 & 1 & 0 & 5 & 1 & 3 \\
24 & 1 & 0 & 0 & 1 & 0 & 0 \\
Total & 144 & 64 & 69 & 65 & 29 & 55 \\
\hline
\end{tabular}




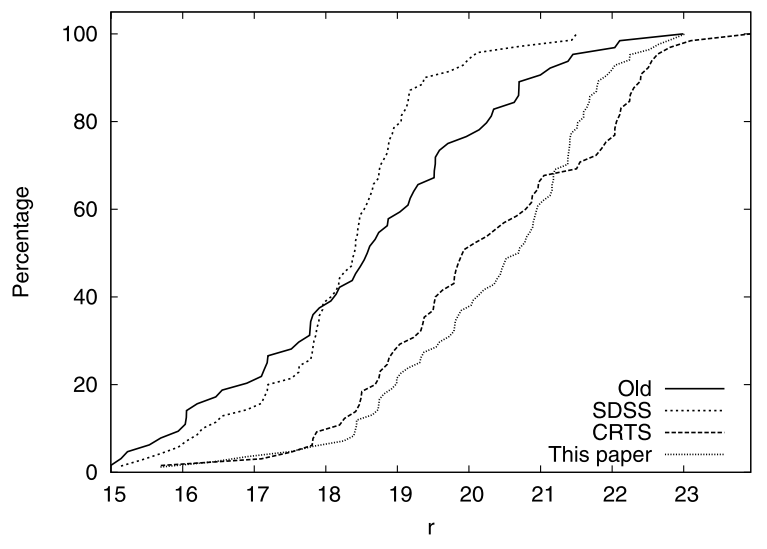

Figure 8. Cumulative distribution of the dwarf novae with SDSS data, according to their $r$ magnitude in quiescence.

subsample as 'old' in the discussion that follows. The fourth column gives the number of dwarf novae from Table 1 for which SDSS-obtained spectroscopy has been published by Szkody et al. (2009, and previous papers in that series), irrespective of whether they were already known before, the fifth column those that were detected in outburst by CRTS (including objects from the 'old' list) and the sixth column the number of known systems recovered in this study. The last column lists the number of new dwarf nova candidates found in this paper, excluding nine with only SDSS data in outburst. Fig. 8 illustrates the normalized cumulative distribution of the quiescent magnitudes of the dwarf novae not discovered by SDSS or CRTS ('old' systems), those detected spectroscopically by SDSS, those found in outburst by CRTS and those detected in this paper (both already-known systems as well as new ones). The number of new systems from CRTS and our study increases dramatically compared to the previously known ones for $r \gtrsim 21$. A Kolmogorov-Smirnov test shows that there is less than 1 per cent probability that the magnitude distribution of the CRTS and our new dwarf novae differ from that of the previously known ones. From the fact that about as many dwarf novae were recovered as new ones found for $r \leq 20$ (21 compared to 19) one may conclude that about half of the regularly outbursting dwarf novae are known with $r \leq 20$ (including recent CRTS discoveries) in the SDSS fields. Outside the SDSS fields, there will be a much smaller fraction of the systems known, as SDSS contributed significantly to the (spectroscopic) identification of dwarf novae. Also, only a small fraction of fainter systems is known. These may be objects that are simply further away, or may belong to a population of intrinsically fainter objects with lower mass transfer rates.

The apparently fainter systems were predominantly discovered by CRTS, as the SDSS spectroscopy does not reach deep enough. Only experiments like CRTS, which are similar in nature to the way the study presented here was set up, are able to discover them at this time. The number of dwarf novae found in this paper decreases significantly for $r>21$ at quiescence, essentially because of the magnitude limit of SDSS and the outburst cut-off at mag 18 used for matching with the astrometric catalogues. CRTS goes deeper than this and still discovers a large number of systems with $r \approx 22$.

The fact that CRTS is discovering mostly faint systems is clearly illustrated in Fig. 9. The top panel compares the distribution of the observed outburst magnitudes of the dwarf novae found by CRTS and those known before, whereas the bottom panel shows the same for the quiescent magnitudes. Note that a minimum magnitude is
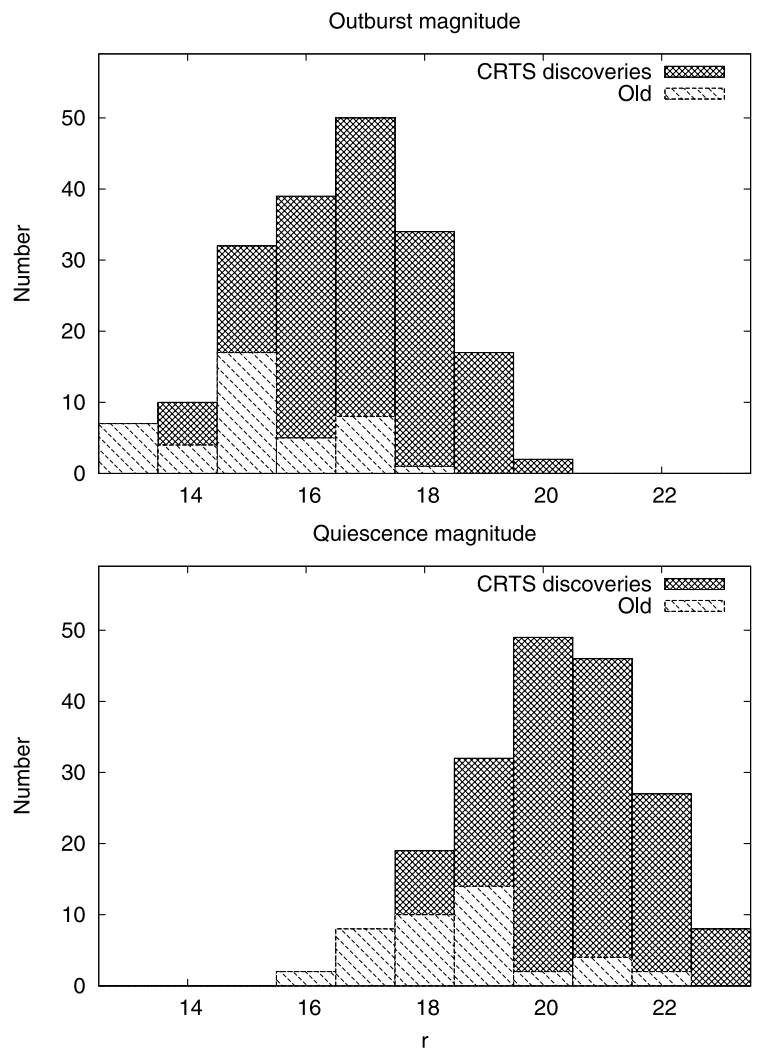

Figure 9. Distribution of previously known and newly discovered dwarf novae detected in outburst by CRTS.

often difficult to obtain for CRTS objects fainter than mag 22, at which level magnitudes can no longer be trusted. For a number of systems, no reliable quiescent magnitude could be obtained, in which case it was set to a default of 23 . Consequently, the outburst amplitudes may be underestimated in these cases. The data are based on CRTS outbursts for 42 previously known dwarf novae and 149 newly discovered systems from the whole sky observed by CRTS (not limited to SDSS fields) in the period of 2007 November (when CRTS operations started) to 2009 May. CRTS detections are not biased towards either new or already known dwarf novae, both are reported in the same way. CRTS did not discover any new dwarf novae with $r \leq 17$ at quiescence.

The outburst amplitude may be a characteristic that reflects differences between 'normal' and intrinsically faint dwarf novae, as WZ Sge systems only show large amplitude superoutbursts. Although the largest outburst amplitude dwarf novae tend to be new CRTS discoveries, the distribution of the amplitudes between previously known and CRTS discovered systems are very much alike (Fig. 10). The new dwarf novae presented in this paper also tend to have larger amplitudes. This is in part due to a selection effect because the constraints used to detect them favour those systems with larger amplitudes. However, as for the CRTS-discovered dwarf novae, the tail of the distribution is also longer than for the previously known systems.

Lacking orbital period information, a difference in dwarf novae populations can also be deduced from a difference in outburst frequency. Our sample of observed outbursts is, however, too fragmentary compared to those of the known systems to draw any firm conclusions. In order to get a better estimate for the outburst 


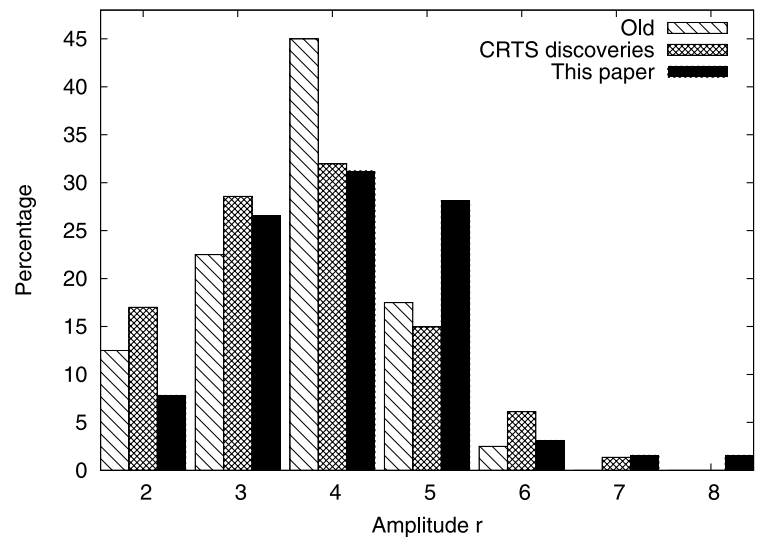

Figure 10. Distribution of the outburst amplitudes of the previously known and newly discovered dwarf novae detected in outburst by CRTS and of the new systems found in this paper.

Table 6. Estimated outburst frequency for confirmed dwarf novae derived from CRTS observations.

\begin{tabular}{crrrr}
\hline $\begin{array}{c}\text { Time in } \\
\text { outburst (per cent) }\end{array}$ & $\begin{array}{r}\text { Previously } \\
\text { known }\end{array}$ & $\begin{array}{r}\text { SDSS } \\
\text { discoveries }\end{array}$ & $\begin{array}{r}\text { CRTS } \\
\text { discoveries }\end{array}$ & $\begin{array}{r}\text { This } \\
\text { paper }\end{array}$ \\
\hline No outburst & 38 & 10 & 0 & 25 \\
$0-5$ & 21 & 10 & 33 & 7 \\
$5-10$ & 22 & 7 & 73 & 7 \\
$10-15$ & 16 & 6 & 24 & 4 \\
$15-20$ & 7 & 1 & 15 & 5 \\
$20-25$ & 3 & 0 & 5 & 0 \\
$25-30$ & 6 & 1 & 2 & 4 \\
$30-35$ & 0 & 1 & 0 & 1 \\
$35-40$ & 0 & 2 & 2 & 0 \\
$40-50$ & 3 & 0 & 2 & 0 \\
$50-100$ & 10 & 1 & 1 & 1 \\
Total & 126 & 39 & 157 & 54 \\
\hline
\end{tabular}

frequency, CRTS data of known systems ${ }^{10}$ were studied and compared to the CRTS data of the new objects. Only light curves with more than 20 data points were considered (i.e. effectively observed on at least five nights). The available data had a time-span from 3 to almost 5 years, and up to 292 data points were available. A dwarf nova was considered to be in outburst if its brightness differed by at least $2 \mathrm{mag}$ from its quiescent value. The number of data points in outburst divided by the total number of observations is then a measure of the outburst frequency. Table 6 lists the outburst frequencies determined in this way for the previously known systems, excluding CRTS and SDSS discoveries (second column), SDSS discovered dwarf novae (third column), CRTS discoveries (fourth column) and the new systems from Table 2 (last column). CRTS discoveries are biased because they had to be in outburst for them to be discovered. But considering only dwarf novae which are observed at least once in outburst by CRTS, the major difference seems to be the large proportion of known systems which are active a large fraction of the time (mostly Z Cam type systems).

\footnotetext{
${ }^{10}$ Available from http://nesssi.cacr.caltech.edu/catalina/CVservice/
}

The previously known systems were more likely to be found in outburst than the new ones. This is the reverse of what is expected as all new systems were found by outbursts, while some of the old ones were not. Considering only the fact whether new and old systems were found to be in outburst by CRTS or not, Fisher's exact test gives only a 3 per cent probability that the same or a more extreme difference is observed for samples of the same population. Still, it is not possible to conclude that the new and old populations are entirely different on the basis of this statistic alone, but there is more than a hint that on average the new systems are not only fainter but also less active. This supports the conclusion from Gänsicke et al. (2009) that deep CV samples contain a significant fraction of intrinsically faint systems with low accretion rates. At first sight, there seems to be no difference in the outburst frequency of the previously known dwarf novae and those discovered by SDSS spectroscopically (e.g. the fraction of systems without outbursts is essentially the same), which may seem a contradiction. However, note that the SDSS sample considered in this paper is only a subset of all CVs found by SDSS, namely those which have had at least one observed outburst. It is very likely that among the remainder of the SDSS CVs there is a significant number of infrequently outbursting systems.

The time CRTS has been in operation is too short to study changes in the rate at which it is discovering new dwarf novae. This could have given an indication of how many systems are still to be discovered. But, both the CRTS discoveries and the new dwarf novae found in this paper suggest a large population of faint dwarf novae of which only a small fraction is known at this time. Detailed studies of this kind will become more feasible in the intermediate future when more CRTS observations are available, and in the longer term through LSST.

\section{CONCLUSION}

In this paper, 64 new CV candidates, mostly dwarf novae, are presented, resulting from data mining between existing photometric and astrometric catalogues. 16 of these systems are unmistakably confirmed as CVs through follow-up spectroscopy. It is shown that a large number of faint CVs are missed by surveys like SDSS, but can be picked up by either transient surveys like CRTS or archival plate studies. The importance of having historical plates available electronically and measured cannot be overestimated in this regard. Even including the recent CRTS discoveries, it is estimated that fewer than half the dwarf novae with a quiescent $r \leq 20$ are currently known in the SDSS fields, and substantially fewer of the fainter ones. It appears that CVs will constitute a significant source of transients for future surveys such as LSST and Pan-STARRS.

Studying amplitude and outburst frequency suggests that the new faint dwarf novae consist of systems both similar to the previously known ones but simply farther away, and of intrinsically fainter systems. The data that will become available in the near future from CRTS and the planned surveys will give an opportunity to study the latter population in much more detail.

Both SDSS J093946.03+065209.4 and SDSS J162900.55+ 341022.0, two of the dwarf novae candidates found, have unusual spectra for CVs and deserve to be studied in more detail.

\section{ACKNOWLEDGMENTS}

This study made use of Simbad and VizieR (Ochsenbein et al. 2000), the USNOFS Image and Catalogue Archive operated by the United States Naval Observatory, Flagstaff Station, optical images generated by the NEAT through the Skymorph website and 
of data provided by the GALEX mission and the SDSS. GALEX is a NASA small explorer, launched in 2003 April. It is operated for NASA by Caltech under NASA contract NAS5-98034. Funding for the SDSS has been provided by the Alfred P. Sloan Foundation, the Participating Institutions, the National Aeronautics and Space Administration, the National Science Foundation, the U.S. Department of Energy, the Japanese Monbukagakusho and the Max Planck Society. The SDSS web site is http://www.sdss.org/. The SDSS is managed by the Astrophysical Research Consortium for the participating institutions. The participating institutions are The University of Chicago, Fermilab, the Institute for Advanced Study, the Japan Participation Group, The Johns Hopkins University, Los Alamos National Laboratory, the Max Planck Institute for Astronomy, the Max Planck Institute for Astrophysics, New Mexico State University, University of Pittsburgh, Princeton University, the United States Naval Observatory and the University of Washington.

The CSS survey is funded by the National Aeronautics and Space Administration under Grant No. NNG05GF22G issued through the Science Mission Directorate Near-Earth Objects Observations Programme. The CRTS survey is supported by the U.S. National Science Foundation under grant No. AST-0909182.

This paper is based in part on observations made with the William Herschel Telescope operated on the island of La Palma by the Isaac Newton Group in the Spanish Observatorio del Roque de los Muchachos of the Instituto de Astrofísica de Canarias and on observations (GN-2008A-Q-86 and GS-2009A-Q-73) obtained at the Gemini Observatory, which is operated by the Association of Universities for Research in Astronomy, Inc., under a cooperative agreement with the NSF on behalf of the Gemini partnership: the National Science Foundation (United States), the Science and Technology Facilities Council (United Kingdom), the National Research Council (Canada), CONICYT (Chile), the Australian Research Council (Australia), Ministério da Ciência e Tecnologia (Brazil) and Ministerio de Ciencia, Tecnologa e Innovación Productiva (Argentina).

We thank Areg Mickaelian for providing us with copies of the paper by Hovanissian $(1978,1982)$. We also thank the referee, Christian Knigge, for his constructive remarks to improve the paper.

\section{REFERENCES}

Abazajian K. et al., 2005, AJ, 129, 1755

Abazajian K. N. et al., 2009, ApJS, 182, 543

Abraham Z., Carrara E. A., 1998, ApJ, 496, 172

Aungwerojwit A. et al., 2006, A\&A, 455, 659

Budavári T. et al., 2009, ApJ, 694, 1281

Copenhagen University Obs., Institute of Astronomy, Cambridge, U., Real Instituto y Observatorio de la Armada en San Fernando, 2006, Carlsberg Meridian Catalog Number 14

Drake A. J. et al., 2009, ApJ, 696, 870

Ducourant C. et al., 2006, A\&A, 448, 1235
Eisenstein D. J. et al., 2006, ApJS, 167, 40

Gänsicke B. T., 2005, in Hameury J.-M., Lasota J.-P., eds, ASP Conf. Ser. Vol. 330, The Astrophysics of Cataclysmic Variables and Related Objects. Astron. Soc. Pac., San Francisco, p. 3

Gänsicke B. T. et al., 2009, MNRAS, 397, 2170

Horne K., 1986, PASP, 98, 609

Hovanissian E. Y., 1978, Soobshcheniya. Byurakan. Astrof. Obs., 50, 5

Hovanissian E. Y., 1982, Soobshcheniya. Byurakan. Astrof. Obs., 53, 77

Howell S. B., Nelson L. A., Rappaport S., 2001, ApJ, 550, 897

Jones D. H. et al., 2005, MNRAS, 355, 747

Jones D. H. et al., 2009, MNRAS, 399, 683

Kolb U., 1993, A\&A, 271, 149

Kolb U., Baraffe I., 1999, MNRAS, 309, 1034

Lasker B. M. et al., 2008, AJ, 136, 735

Lasota J.-P., 2001, New Astron. Rev., 45, 449

Lawrence A. et al., 2007, MNRAS, 379, 1599

Li N., Thakar A. R., 2008, Comput. Sci. Eng., 10, 18

Lohmann W., 1949, Astron. Nachrichten, 277, 37

Marsh T. R., 1989, PASP, 101, 1032

Martin D. C. et al., 2005, ApJ, 619, L1

Meyer F., Meyer-Hofmeister E., 1984, A\&A, 132, 143

Monet D. G. et al., 2003, AJ, 125, 984

Ochsenbein F., Bauer P., Marcout J., 2000, A\&AS, 143, 23

Osaki Y., 1996, PASP, 108, 39

Pesch P., Sanduleak N., 1987, Inf. Bull. Var. Stars, 2989

Pretorius M. L., Knigge C., 2008, MNRAS, 385, 1485

Pretorius M. L., Knigge C., Kolb U., 2007, MNRAS, 374, 1495

Richards G. T. et al., 2002, AJ, 123, 2945

Ritter H., Kolb U., 2003, A\&A, 404, 301

Szkody P. et al., 2004, AJ, 128, 1882

Szkody P. et al., 2009, AJ, 137, 4011

Voges W. et al., 1999, A\&A, 349, 389

Warren S. J. et al., 2007, MNRAS, 375, 213

Welsh B. Y. et al., 2005, AJ, 130, 825

Willems B., Kolb U., Sandquist E. L., Taam R. E., Dubus G., 2005, ApJ, 635,1263

\section{SUPPORTING INFORMATION}

Additional Supporting Information may be found in the online version of this article:

Table 1. Known dwarf novae with SDSS ugriz data.

Table 3. Observed outbursts of the new dwarf novae.

Please note: Wiley-Blackwell are not responsible for the content or functionality of any supporting materials supplied by the authors. Any queries (other than missing material) should be directed to the corresponding author for the article.

This paper has been typeset from a $\mathrm{T}_{\mathrm{E}} \mathrm{X} / \mathrm{LT}_{\mathrm{E}} \mathrm{X}$ file prepared by the author. 